the hypothalamic-pituitary hormones and the gonadal steroids in both men and women. The remainder of the book describes the classification, investigation and treatment of functional infertility. Inexperienced readers may not realise that the classifications given are not in universal use and that the account of anovulatory infertility is incomplete - the polycystic ovary syndrome is not mentioned and there is no discussion of weight-related amenorrhoea. Relatively little space is given to hyper-prolactinaemia and its management with bromocriptine and disproportionately much to the use of human menopausal gonadotrophins. Admittedly, the latter presents more difficulties in practice but the former is necessary more often. Only those involved in research will be interested in the effects of compounds such as RO 8347 and Epimestrol. The section on male infertility is valuable and suggests that the logical investigation of male infertility is becoming possible.

In fact, this book is not meant to be a comprehensive textbook but an account of the views and work of a team of distinguished Israeli gynaecological endocrinologists. As such it is of interest to all who work in the same field.

\section{Fundamentals of Oncology}

By Henry C. Pitot. Pp. 192, illustrated. Marcel Dekker, New York and Basel, 1978. Sw. Fr. 26.00.

The student of biology will, at some time in his course, be stimulated to enquire about growth and about those patterns of disordered growth, cancers or neoplasms. He will find in this book clear accounts of many aspects of cancer, its aetiology, biology, biochemistry and immunology drawn together in a stimulating and informative way.

This book is not an over-simplified account for lay people. Each chapter has a good introduction and is well referenced. A very readable introduction to terms used in describing neoplastic disease is given so that the biologist or clinician who has not specialized in cancer will find no difficulty later. Once the terminology is mastered, the aspirations and frustrations of those working in the field of cancer, whether it be the biochemistry of the cell or the immunology of the disease, is presented as a fascinating story.

When the current hypotheses and overall results are examined in this way the reader may well be a little despondent. Nevertheless, whether he is a student or a professor, he will have, as a result of reading the book, a much better idea of the problems facing those working on neoplastic disorders.

\section{Gastrointestinal Motility in Health and Disease}

Edited by H. L. DuthIE. Proceedings of the 6th International Symposium on Gastrointestinal Motility held at the Royal College of Surgeons of Edinburgh, September 1977. Pp. 684, illustrated. MTP Press, Lancaster, 1978. £15.95.

This book reports the sixth International Symposium on Gastrointestinal Motility held at the Royal College of Surgeons of Edinburgh during September 1977. W. Silber in the 'Workshop' section believes the statement by Bayliss and Starling in 1899 'that on no subject in physiology do we meet with so many discrepancies of fact and opinion as in that of physiology of the intestinal movement' holds true today. Whether this is so or not, this volume brings together more than 70 papers (with discussion) in 14 sections from international experts in gastrointestinal motility. The volume is rather daunting for the practising clinician, but disordered motility may well be one of the commonest abnormalities causing gastrointestinal symptoms and these patterns may eventually prove characteristic of disorders such as the irritable colon syndrome. Particularly interesting are the sections on migrating motor complexes, especially the studies in man, intestinal polypeptides, the effect of diet on motility, method of analysis, clinical aspects of motility and lower oesophageal sphincter control. The clinician will want to refer to selected sections in the book but the physiologist, physicist and pharmacologist will need to keep it.

\section{Hepatotrophic Factors}

Ciba Foundation Symposium 55 (new series). Edited by Ruth Porter and Julie Whelan. Pp. 405, illustrated. Elsevier, Amsterdam, Oxford, New York; Excerpta Medica, North Holland, 1978. \$36.00.

The book is a collection of papers presented at a recent symposium by an impressive number of international experts. The title is somewhat unfortunate since there seems to be no agreement between the contributors of a definition for the term 'hepatotrophic factors'. To some authors this implies hepatocyte hypertrophy, to others hyperplasia, and to yet others both of these. Weinbren clearly shows that the 2 processes are often quite distinct. The contributions are chiefly concerned with the mechanisms involved in the proliferation of liver cells after experimental partial hepatectomy and the atrophy occurring after diversion of portal blood from the liver. Several groups show the importance of insulin for the former although this does not initiate hepatocyte proliferation, the evidence for glucagon being less convincing. Epidermal growth factor may be an important initiating factor and its effect is certainly augmented by insulin. However, Junge and Creutzfeldt show that the maximal increase in DNA synthesis occurs at a time when portal and systemic blood levels of insulin are reduced. The liver atrophy following portal blood diversion is in many respects the opposite to the regeneration following partial hepatectomy. Once again insulin, which is present in very high concentrations in portal blood, appears to be important in preventing this, but Starzl shows that non-pancreatic splanchnic blood factors may also be involved. Whether or not these findings have any immediate clinical application is not clear. Bucher showed that insulin and glucagon infusions improved survival in murine hepatitis, but Junge and Creutzfeldt could not confirm this for carbon tetrachlorideinduced hepatic necrosis in the rat and systemic blood levels for these hormones are already high in human fulminant hepatic failure.

Other contributions include studies on isolated hepatocytes and the value of portacaval shunts for glycogen storage diseases and congenital hyperlipidaemias. The discussions following each paper are obviously lively, but clearly indicate our enormous lack of knowledge in this potentially important field.

\section{Hip Disorders in Children}

By G. C. Lloyd-Roberts and A. H. C. Ratliff. Postgraduate Orthopaedics Series - General. Editor: A. G. APLEY. Pp. 291, illustrated. Butterworths, London, Boston etc., 1978. $£ 17.50$.

This is an excellent and important book written by 2 experts on hip disorders in children. It is a specialized subject and this comprehensive work will be important for orthopaedic consultants and also for trainees. The presentation of the material is very good indeed, and although many of the $\mathrm{X}$-rays do leave much to be desired it is recognized that paediatric radiology is extremely difficult and it is important to make allowances for this.

Details on the management of congenital dislocation of the hip are extensively reviewed by $\mathrm{Mr}$ Lloyd-Roberts and with this guide every eventuality in $\mathrm{CDH}$ treatment has been covered satisfactorily. His review of congenital femoral deficiency is also masterly but there is probably overemphasis on the operative aspects of this difficult condition. 
Perthe's disease is again a difficult subject and there has been much progress recently in the classification of the hip 'at risk'. The classification and grading is well surveyed by the authors and a modern treatment plan is outlined. Fractures of the femoral neck in childhood together with slipped upper femoral epiphysis are extensively covered by Mr Ratliff. $\mathrm{He}$ has also beautifully outlined and illustrated miscellaneous hip problems in childhood and the combined authors have produced a classic work which is very welcome and much overdue. The book is highly recommended.

\section{The Nails in Disease}

By Peter D. Samman. Third Edition. Pp. 197, illustrated. William Heinemann Medical Books, London, 1978 $£ 10.00$.

Already a classic, this elegant treatise has no peer. This third edition enjoys a similar format to the others, but it is just that little bit plumper, stuffed more fully with up-to-date succinct information, and has a greater profusion of pictures.

One must guess that for most of the profession, their knowledge of nails and nail diseases could be tucked into a fairly small mental pigeon hole and their views concerning the significance of disorder of these unobtrusive organs would range from zero to an unprintable comment. Appendix II, thus, might come as a surprise wherein is listed that out of 1100 patients barely 350 suffered from either superficial fungal infections or psoriasis. What of the rest, therefore? Likewise appendix I, the Glossary, will come as a distinct shock to medical men and classicists alike - what can 'agnail' and 'onychoheterotropia' mean? - enough to give anyone onychalgia nervosa and drive him into a frenzy of onychotillomania.

The first chapter of the total of 12, Anatomy and Physiology, fully but laconically, with the aid of excellent line drawings, photomicrographs and radiographs explains the fundamentals so that chapter 2, Principal Nail Symptoms, also admirably explicit, is entirely intelligible and they both, in a mere 25 pages, serve as a marvellous introduction to disease.

Psoriasis takes pride of place, and then fungus infection but, despite the 33 illustrations which these chapters share, and the simple and unambiguous text, doctors will continue to muddle their diagnoses and prescribe the costly fungal antibiotic griseofulvin inappropriately for these conditions and for the nail dystrophy associated with chronic paronychia, the subject of the next chapter. Then a chapter that deals with the 2 other very common red, scaly, dermatoses, dermatitis and lichen planus, and the nail changes of alopecia areata and some dermatological oddities. Acquired Nail Disorders is the next chapter, and these range from infective, pustular but non-infective, dramatic but unimportant, and various. General physicians will be interested in chapters 8 , Impaired Peripheral Circulation, and 9, General Medical Conditions, and will be able to find out what Terry actually did describe, when Beau named his lines, and why the yellow nail syndrome, described by the author in 1964, is of importance to the patient's system.

Chapter 10, Trauma, the causes ranging from nail varnish, nail biting, nail picking, and nail bashing by footwear, accounts for a large proportion of the nail dystrophies that bring the patient to the doctor. Tumours, Chapter 11, benign ones notably warts, and curious ones such as subungual exostoses, Developmental Abnormalities, Chapter 12, and some of these one would scarcely credit as genuine and, like the giraffe had one not seen an example one would not believe it, completes a volume which every dermatologist who is not blessed with an earlier edition, must own and every medical library must buy.
Occurrence, Diagnosis and Sources of Hospital-Associated Infections (Handbook on Hospital-Associated Infections, Vol. 1)

Edited by W. J. Fahlberg and D. Gröschel. Pp. 138, hard cover illustrated. Marcel Dekker Inc., New York and Basel, 1978. Sw. fr. 45.00.

This book offers a useful general framework for its important subject, its fundamental defect is that it 'is aimed at the broadest spectrum of health care personnel ... including individuals in central services, housekeeping, dietary, and laundry'. This is of course an impossible target. It could have been reached only in the non-technical opening section on the historical background; but this is too brief and stops, unaccountably, in the 1880s. Experienced teachers of this subject know that their students can only achieve adequate perspective by appreciating the fascinating evolutionary changes that have occurred continuously right up to the present day in hospitals, patients, and micro-organisms alike.

In its 4 main sections the book concisely reviews the clinical, laboratory, therapeutic and aetiological aspects of hospital infection. Here the reader is constantly aware of an uneasy balance between the technical discussion that is required by a medical audience and the simpler approach sought by other groups. Junior clinical students would undoubtedly obtain from this book a helpful impression of the many complex problems now posed by infections in our hospitals. Unfortunately, however, the audience who could gain most from the book is the least likely to be able to afford it. Although cheaply printed, the slim volume is highly priced in Swiss francs. Its cost in terms of weaker currencies is at least twice as much as for equivalent indigenous books.

Physiology of the Eye. An Introduction to the Vegetative Functions

By Irving Fatr. Pp. viii + 232, illustrated. Butterworthş Boston and London, 1978. £13.00 U.S. \$26.00.

The curious subtitle An Introduction to the Vegetative Functions seems inapt on the basis of the definition of vegetative as 'to live like a vegetable or to live an inactive almost purely physical, or dull life'. Professor Irving Fatt writes on the physiology of the eye in a manner which makes the subject seem anything but a dull life. Here is a book which fills a most useful gap in the literature of physiology. In the preface the author correctly comments that he could not find an introductory textbook to the physiology of the eye and has based his book on lecture notes given at the University of California in Berkeley. By vegetative physiology he means the biophysical and biochemical processes that maintain the eye in its normal state.

The book is divided into successive chapters on Anatomy, The Aqueous Humor, The Intra-ocular Pressure, The Vitreous Body, The Lens, The Cornea, The Sclera, The Retina and The Tears and Lids. This arrangement deliberately corresponds to the arrangement in Davson's The Ej'e so that readers may use the two books to complement each other (also a deserved compliment to Hugh Davson's book). The Aqueous and the Cornea alone form more than half the 222 pages of the book which reflects the knowledge of physiology in these subjects. There is much excellent and concise information in these chapters and there are in particular many justified references to D. M. Maurice's work on corneal physiology. The illustrations, all in black and white, are well drawn and liberally used. In particular the measurement and physiology of intra-ocular pressure are dealt with in detail which reflects the usefulness of this book to optometric students. The chapters on the Vitreous and the Lens are somewhat sparse which is a disappointment as there has been much useful work on these topics in recent years which could have been included. The Sclera warrants

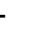

